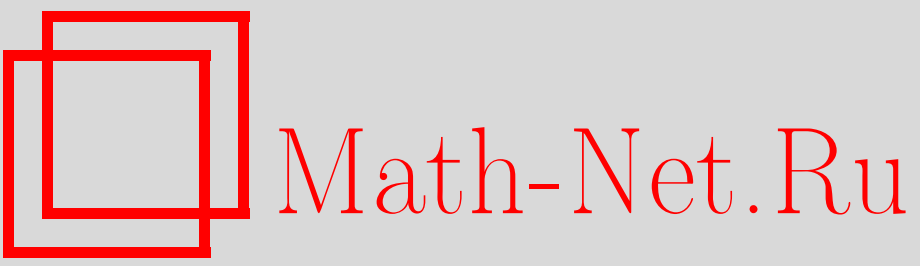

А. Н. Колмогоров, Математические модели турбулентного движения несжимаемой вязкой жидкости, УМН, 2004, том 59, выпуск 1, 5-10

DOI: https://doi.org/10.4213/rm697

Использование Общероссийского математического портала Math-Net.Ru подразумевает, что вы прочитали и согласны с пользовательским соглашением

http://www.mathnet.ru/rus/agreement

Параметры загрузки:

IP : 54.224 .60 .19

26 апреля 2023 г., 14:52:16 


\title{
МАТЕМАТИЧЕСКИЕ МОДЕЛИ ТУРБУЛЕНТНОГО ДВИЖЕНИЯ НЕСЖИМАЕМОЙ ВЯЗКОЙ ЖИДКОСТИ
}

\author{
А. Н. Колмогоров
}

СОДЕРЖАНИЕ

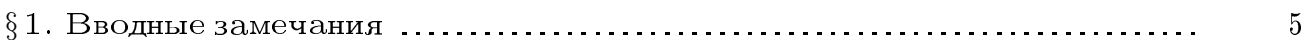

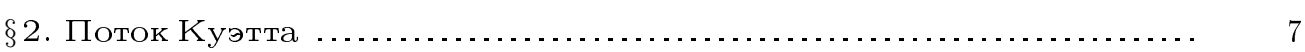

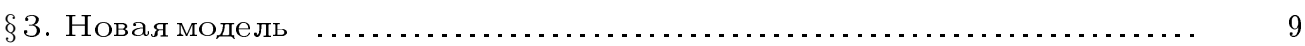

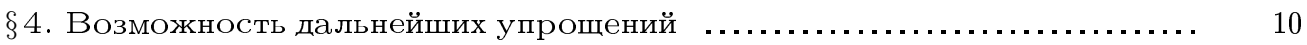

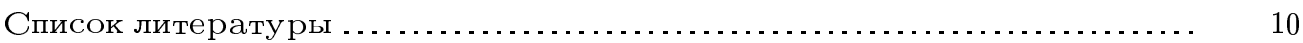

\section{$\S$ 1. Вводные замечания}

1.1. Хотя реальная турбулентность всегда существенно трехмерна, наши модели будут двумерными. Будет рассматриваться движение в ограниченной области, хотя эта ограниченность и будет создаваться искусственно при помоши постулирования периодичности. Эти ограничения позволяют считать нужные теоремы существования и единственности установленными (Ладыженская [1]): дело идет действительно об изучении динамической системы в надлежащем функциональном фазовом пространстве. Практическая уверенность в том, что при этом типичные свойства реальной турбулентности не будут утеряны, подтверждается вычислительным экспериментом [2].

1.2. Реальные факты, лежашие в основе наших рассмотрений, таковы.

А) При уменьшении вязкости $\nu$ стационарные (ламинарные) решения стационарных задач становятся обычно или (A1) неустойчивыми, или (А2) столь мало устойчивыми, что реально не наблюдаются. Реально наблюдаемые (турбулентные) решения, завися сложным образом от времени, подчиняются статистическому стационарному режиму, не зависящему практически от начального положения.

В) При стремлении вязкости к нулю практически наблюдаемые решения становятся все менее гладкими. За счет такой изрезанности профиля скоростей дис-

Статья была, по-видимому, написана в конце пятидесятых годов. Текст носит окончательный характер, но список литературы не детализирован. Я. Г. Синай добавил к тексту несколько примечаний и включил в список литературы ряд названий, которые отвечают содержанию статьи. 
сипация на единицу массы

$$
\frac{1}{2} \nu \sum_{i, j}\left(\frac{\partial u_{i}}{\partial x_{j}}+\frac{\partial u_{j}}{\partial x_{i}}\right)^{2}
$$

даже в отдалении от стенок остается порядка

$$
\frac{U^{3}}{L}
$$

где $U$ - типичная скорость, а $L$ - типичная длина.

Явления типа А хорошо изучены в динамических системах с конечньм числом степеней свободы. При одной степени свободы (фазовое пространство двумерно) это (А1) превращение устойчивой неподвижной точки 0 в неустойчивую с наматыванием траекторий, начинаюшихся вблизи 0, на предельньй цикл:

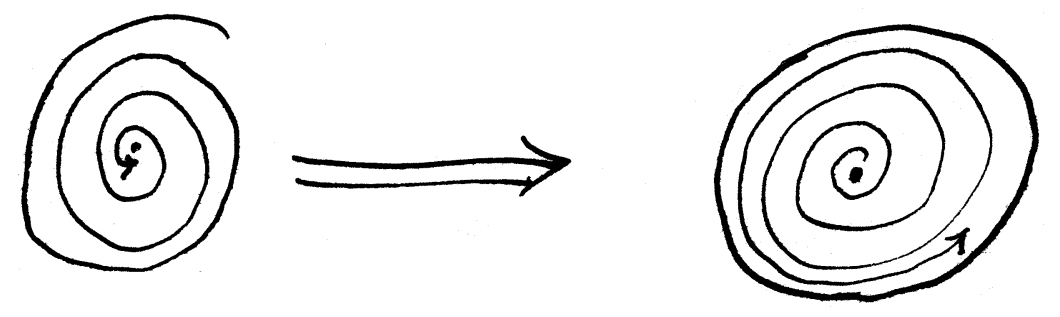

или (А2) стягивание к 0 маленького цикла, за пределами которого траектории наматьваются на большой цикл ${ }^{1}$ :
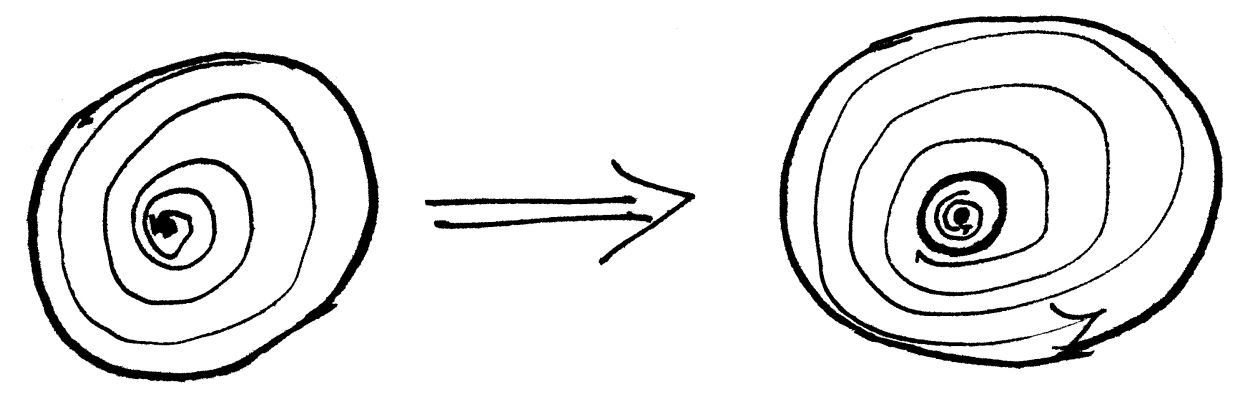

Явления типа В изучены лишш на уровне полуэмпирических теорий, приведших уже к большому ряду качественных и количественных результатов, хорошо оправдьвающихся экспериментально.

В частности, по-видимому, вообще говоря, при любом $\varepsilon>0$ порядок малости разностей скоростей $\Delta u$ на расстоянии $r$ остается меньше $r^{1 / 3-\varepsilon}$ :

$$
\frac{\Delta u}{r^{1 / 3-\varepsilon}} \rightarrow 0 \text { при } r \rightarrow 0
$$

равномерно относительно $\nu$. Таким образом, предельньй режим при $\nu=0$ следует представлять себе без разрьвов для скоростей (см. [3]).

\footnotetext{
${ }^{1}$ Для явлений такого типа исползуются термины “бифуркация Андронова”, “бифуркация Андронова-Хопфа", "бифуркация Хопфа". Подобные бифуркации происходят также в многочисленных системах и достаточно хорошо изучены. (Прим. Я. Г. Синая.)
} 


\section{$\S$ 2. Поток Куэтта}

Явления типа (А2) можно ожидать с достаточной уверенностью в обстановке, которую мы сейчас опишем. В полосе плоскости $(x, y)$, ограниченной прямыми $y= \pm a / 2$, движение подчинено уравнениям ${ }^{2}$

$$
\left.\begin{array}{l}
\frac{D u}{D t}=-\frac{\partial p}{\partial x}+\nu \Delta u \\
\frac{D v}{D t}=-\frac{\partial p}{\partial y}+\nu \Delta v \\
\frac{\partial u}{\partial x}+\frac{\partial v}{\partial y}=0
\end{array}\right\}
$$

На прямых $y= \pm a / 2$

$$
u= \pm u_{0}, \quad v=0
$$

Реальные явления между двумя движущимися плоскостями можно, по-видимому, имитировать достаточно хорошо, ограничившись функциями $u, v, p$, периодическими по $x$ с достаточно большим периодом $2 b$. Качественно та же картина получится, вероятно, и при любом $b$, например, $b=a$.

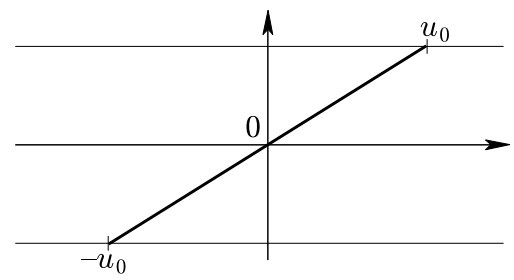

Ламинарное решение

$$
u=\frac{2}{a} u_{0} y
$$

всегда устойчиво (см. [4]), но устойчивость его при малом $\nu$, по-видимому, чрезвычайно слаба*. Турбулентньй профиль средних скоростей $\bar{u}$ должен иметь вид

2 Здесь $\frac{D}{D t}$ означает субстанциональную производную по времени (см.: А. Н. Колмогоров. Уравнения турбулентного движения несжимаемой жидкости // Изв. АН СССР. Сер. физ. 1942. Т. 6, № 1-2. С. 56-58; Избр. труды. Математика и механика. М.: Наука, 1985. С. 294-296; А. С. Монин, А. М. Яглом. Статистическая гидромеханика. Ч. 1. М.: Наука, 1965, гл. III, раздел 6.2). (Прим. ред.)

*Напомним, что бесконечно малые возмущения $\varphi$,

$$
\psi=\psi_{0}+\varphi
$$

при $\psi_{0}$, зависящем только от $y$, подчиняются уравнению 〈Орра-Зоммерфелшда - Я. Г. Синай

$$
\frac{\partial}{\partial t} \Delta \varphi-\frac{\partial \varphi}{\partial x} \psi_{0}^{\mathrm{iii}}+\psi_{0}^{\prime} \frac{\partial}{\partial x} \Delta \varphi=\nu \Delta \Delta \varphi
$$




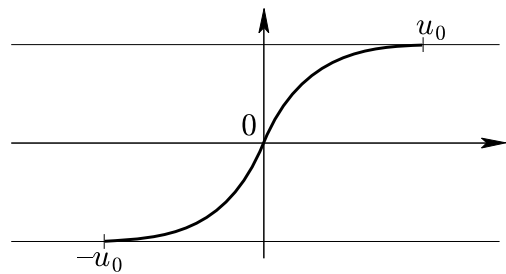

При $\nu \rightarrow 0$ он вырождается в

$$
u= \begin{cases}-u_{0}, & y=-a / 2, \\ 0, & -a / 2<y<a / 2, \\ u_{0}, & y=a / 2\end{cases}
$$

Однако из полуэмпирической формулы ${ }^{3}$

$$
\frac{u_{0}}{u_{\tau}}=A+B \log \frac{a u_{\tau}}{\nu}
$$

вытекает, что, положив

$$
u_{0}=B \log \frac{1}{\nu}
$$

получим при $\nu \rightarrow 0$

$$
u_{\tau} \rightarrow 1
$$

По обычным полуэмпирическим конщепциям, основанньм на идеях Кармана, статистический режим решения при $\nu \rightarrow 0$ и условии (3) должен стремиться к определенному предельному невырожденному режиму равномерно в любой полосе

$$
-\frac{a}{2}+\varepsilon<y<\frac{a}{2}-\varepsilon
$$

Вероятно, это так и в строгом математическом смысле, но, может быть, при несколько отличной функции

$$
u_{0}=f(\nu),
$$

близкой к (3) лишш в смысле

$$
\frac{\log f(\nu)}{\log \log (1 / \nu)} \rightarrow 1
$$

Так как

$$
\int_{0}^{b} v(x, y) d x=0
$$

то законно положить

$$
u=\frac{\partial \psi}{\partial y}, \quad v=-\frac{\partial \psi}{\partial x}
$$

\footnotetext{
${ }^{3}$ В этом тексте А. Н. Колмогоров не объясняет смысла переменной $\tau$ и происхождение выписьваемой ниже полуэмпирической формулы. Судя по другим материалам из его архива, $\tau$ есть безразмерная вертикальная координата, $\tau=\nu \frac{\partial \bar{u}}{\partial y}-p b_{x y}$, где $p$ - давление, $b_{x y}=\overline{u^{\prime} v^{\prime}}$. (Прим. Я.Г. Синая.)
} 
где $\psi$ периодично по $x$ с периодом $b$. Для $\psi$ получим (выгитая из $\partial / \partial y$ от первого уравнения (1) $\partial / \partial x$ от второго)

$$
\frac{\partial}{\partial t} \Delta \psi-\frac{\partial \psi}{\partial x} \frac{\partial}{\partial y} \Delta \psi+\frac{\partial \psi}{\partial y} \frac{\partial}{\partial x} \Delta \psi=\nu \Delta \Delta \psi
$$

Ограничиваясь решениями с

$$
\int_{-a / 2}^{a / 2} u(x, y) d y=0
$$

и замечая, что $\psi$ сушественно лишш с точностью до константы, можно заменить краевые условия $\left(1^{\prime}\right)$ на

$$
\psi=0, \quad \frac{\partial \psi}{\partial y}= \pm u_{0}
$$

при $y= \pm a / 2$.

При конечном $\nu$ основная задача заключается в изучении инвариантных по отношению к (4) мер в пространстве $\psi(x, y)$, подчиненных $\left(4^{\prime}\right)$.

\section{§. Новая модель}

Изучение предельного поведения инвариантных мер при $\nu \rightarrow 0$ затруднительно в обстановке $\S 2$ ввиду неясности с разумным выбором функции $\left(3^{\prime}\right)$. Вместо этого можно рассмотреть решения системы

$$
\left\{\begin{array}{l}
\frac{D u}{D t}=-\frac{\partial p}{\partial x}+\nu \Delta u+\gamma \sin \left(\frac{\pi}{a} y\right) \\
\frac{D v}{D t}=-\frac{\partial p}{\partial y}+\nu \Delta v \\
\frac{\partial u}{\partial x}+\frac{\partial v}{\partial y}=0
\end{array}\right.
$$

с периодами $2 a$ и $2 b$ соответственно по $y$ и $x$ и условиями

$$
\int_{-a}^{+a} u(x, y) d y=\int_{-b}^{+b} v(x, y) d x=0 .
$$

Ламинарное решение здесь имеет вид

$$
u=\frac{\gamma}{\nu} \sin \frac{\pi}{a} y
$$

Так как (6) имеет точки перегиба, то возможно, что здесь мы имеем первый пример плоской задачи, для которой без большого труда удается с полной строгостью установить неустойчивость ${ }^{4}$. Во всяком случае турбулентные решения (в смысле нетривиальной инвариантной меры) должны появиться при малых $\nu$. Уравнение (4) преврашается в

$$
\frac{\partial}{\partial t} \Delta \psi-\frac{\partial \psi}{\partial x} \frac{\partial}{\partial y} \Delta \psi+\frac{\partial \psi}{\partial y} \frac{\partial}{\partial x} \Delta \psi=\nu \Delta \Delta \psi+\frac{\pi \gamma}{a} \cos \left(\frac{\pi}{a} y\right) .
$$

\footnotetext{
${ }^{4}$ Это было в действительности сделано в нашей работе с Л. Д. Мешалкиным "Исследование устойчивости стационарного решения одной системы уравнений плоского движения несжимаемой вязкой жидкости", Прикладная математика и механика, 1961, т. 25, № 6, с. 1140-1143. (Прим. Я. Г. Синая.)
} 
Вместо краевых условий $\left(4^{\prime}\right)$ требуется лишш периодичность $\psi$ по $x$ и по $y$ и (для устранения произвольной константы) равенство среднего значения $\psi$ нулю.

В направлении В основная гипотеза заключается в том, что турбулентные инвариантные меры $\mu_{\nu}$ в пространстве $\psi(x, y)$ сходятся при $\nu \rightarrow 0$ к предельной мере $\mu$, сосредоточенной на непрерывных функциях, обладаюших описанными в конще $\S 2$ свойствами.

\section{$\S$ 4. Возможность дальнейших упрощений}

Представляется менее ясньм, какие из намеченных эффектов можно получить для упрощенных уравнений, например, для уравнений пограничного слоя

$$
\begin{gathered}
\left\{\begin{array}{l}
\frac{\partial u}{\partial t}+u \frac{\partial u}{\partial x}+v \frac{\partial u}{\partial y}=-\frac{\partial p}{\partial x}+v \frac{\partial^{2} u}{\partial y^{2}}+F(y), \\
\frac{\partial p}{\partial y}=0 \\
\frac{\partial u}{\partial x}+\frac{\partial v}{\partial y}=0
\end{array}\right. \\
\frac{\partial}{\partial t} \frac{\partial^{2} \psi}{\partial y^{2}}-\frac{\partial \psi}{\partial x} \frac{\partial^{3} \psi}{\partial y^{3}}+\frac{\partial \psi}{\partial y} \frac{\partial^{3} \psi}{\partial x \partial y^{2}}=\nu \frac{\partial^{4} \psi}{\partial y^{4}}+F^{\prime}(y)
\end{gathered}
$$

или предлагавшегося Бюргерсом уравнения ${ }^{5}$

$$
\frac{\partial u}{\partial t}+u \frac{\partial u}{\partial y}=\nu \frac{\partial^{2} u}{\partial y^{2}}+F(y) .
$$

При конечном $\nu$ (но не при изучении предельного перехода $\nu \rightarrow 0$ ) можно построить удовлетворительные конечномерные модели, заменяя (1), (4), (8), (9) уравнениями, дифференщиальными по $t$, но конечно-разностньми по $x$ и $y$, или сохраняя в разложении в ряд Фурье по $x$ и $y$ лиш конечное число членов. Инвариантные турбулентные меры $\mu_{\nu}$ здесь, вероятно, окажутся уже вполне доступньми изучению.

\section{СПИСОК ЛИТЕРАТУРЫ}

[1] О.А. Ладыженская. Докл. РАН (в печати). (См.: О. А. Ладыженская. Математические вопросы динамики вязкой несжимаемой жидкости. М.: Физматгиз, 1961. - Я. Г. Синай.)

[2] (См.: У. Фриш. Турбулентность. Наследие А.Н. Колмогорова. М.: Фазис, 1998. Я. Г. Синай.)

[3], [4] (См.: Цзя-Цзяо Линь. Теория гидродинамической устойчивости. М.: ИЛ, 1958. Я. Г. Синай.)

Поступила в редакцию 01.08 .2003

\footnotetext{
${ }^{5}$ По этому поводу см. работу В. И, К. Ханина, А. Мазеля и Я. Г. Синая "Invariant measures for Burgers equation with stochastic forcing”, Annals of Mathematics (2), 2000, v. 151, № 3, p. 877-960. (Прим. Я.Г. Синая.)
} 